\title{
Efficacy, safety and population pharmacokinetics of sapropterin in PKU patients < 4 years: results from the SPARK open-label, multicentre, randomized phase Illb trial
}

Ania C. Muntau ${ }^{1 *}$, Alberto Burlina ${ }^{2}$, François Eyskens ${ }^{3}$, Peter Freisinger ${ }^{4}$, Corinne De Laet $^{5}$, Vincenzo Leuzzi ${ }^{6}$, Frank Rutsch ${ }^{7}$, H. Serap Sivri ${ }^{8}$, Suresh Vijay ${ }^{9}$, Milva Orquidea Bal ${ }^{10}$, Gwendolyn Gramer ${ }^{11}$, Renata Pazdírková ${ }^{12}$, Maureen Cleary ${ }^{13}$, Amelie S. Lotz-Havla ${ }^{14}$, Alain Munafo ${ }^{15}$, Diane R. Mould ${ }^{16}$, Flavie Moreau-Stucker ${ }^{17}$ and Daniela Rogoff ${ }^{17}$

\begin{abstract}
Background: Sapropterin dihydrochloride, a synthetic formulation of $\mathrm{BH}_{4}$, the cofactor for phenylalanine hydroxylase (PAH, EC 1.14.16.1), was initially approved in Europe only for patients $\geq 4$ years with $\mathrm{BH}_{4}$-responsive phenylketonuria. The aim of the SPARK (Safety Paediatric efficAcy phaRmacokinetic with Kuvan ${ }^{\circledR}$ ) trial was to assess the efficacy (improvement in daily phenylalanine tolerance, neuromotor development and growth parameters), safety and pharmacokinetics of sapropterin dihydrochloride in children $<4$ years.

Results: In total, 109 male or female children <4 years with confirmed $\mathrm{BH}_{4}$-responsive phenylketonuria or mild hyperphenylalaninemia and good adherence to dietary treatment were screened. 56 patients were randomly assigned (1:1) to $10 \mathrm{mg} / \mathrm{kg} /$ day oral sapropterin plus a phenylalanine-restricted diet or to only a phenylalanine-restricted diet for 26 weeks ( 27 to the sapropterin and diet group and 29 to the diet-only group; intention-to-treat population). Of these, 52 patients with $\geq 1$ pharmacokinetic sample were included in the pharmacokinetic analysis, and 54 patients were included in the safety analysis. At week 26 in the sapropterin plus diet group, mean phenylalanine tolerance was 30.5 (95\% confidence interval 18.7-42.3) $\mathrm{mg} / \mathrm{kg} /$ day higher than in the diet-only group $(p<0.001)$. The safety profile of sapropterin, measured monthly, was acceptable and consistent with that seen in studies of older children. Using nonlinear mixed effect modelling, a one-compartment model with flip-flop pharmacokinetic behaviour, in which the effect of weight was substantial, best described the pharmacokinetic profile. Patients in both groups had normal neuromotor development and stable growth parameters.
\end{abstract}

Conclusions: The addition of sapropterin to a phenylalanine-restricted diet was well tolerated and led to a significant improvement in phenylalanine tolerance in children $<4$ years with $\mathrm{BH}_{4}$-responsive phenylketonuria or mild hyperphenylalaninemia. The pharmacokinetic model favours once per day dosing with adjustment for weight. Based on the SPARK trial results, sapropterin has received EU approval to treat patients $<4$ years with $\mathrm{BH}_{4}$-responsive phenylketonuria.

Trial registration: ClinicalTrials.gov, NCT01376908. Registered June 17, 2011.

Keywords: Sapropterin, Phenylalanine hydroxylase, Phenylketonuria, Hyperphenylalaninemia, Pharmacokinetics, SPARK

\footnotetext{
* Correspondence: muntau@uke.de

'University Children's Hospital, University Medical Center Hamburg

Eppendorf, Martinistrasse 52, D-20246 Hamburg, Germany

Full list of author information is available at the end of the article
} 


\section{Background}

Hyperphenylalaninemia (HPA) is a rare inherited metabolic disorder caused by reduced activity of the hepatic enzyme phenylalanine hydroxylase (PAH, EC 1.14.16.1), which catalyses the conversion of phenylalanine (Phe) to tyrosine. Most cases of HPA (98\%) in North American and European populations are due to mutations in the $P A H$ gene but, in rare cases of HPA (1-2\%), the cause can be a defect in the metabolism of the natural PAH cofactor, the $\mathrm{R}$ diastereoisomer of tetrahydrobiopterin $\left(\mathrm{BH}_{4}\right)$ [1-3]. Owing to the reduced activity of PAH due to either mechanism, patients with HPA have an accumulation of Phe in the blood and body tissues and a relative deficiency of tyrosine and subsequent metabolites such as epinephrine [4, 5].

HPA can present with a spectrum of phenotypes that can be grouped into three main categories according to blood Phe concentrations before therapeutic intervention: classical PKU (Phe $>1200 \mu \mathrm{mol} / \mathrm{L}$ ); mild PKU (Phe 600$1200 \mu \mathrm{mol} / \mathrm{L}$ ); mild HPA (Phe 120-600 $\mu \mathrm{mol} / \mathrm{L}$ ) $[2,5,6]$. PKU can lead to cognitive impairment and, if untreated, patients can develop mild-to-severe intellectual disability and other neurological sequelae $[2,5,7]$.

The therapeutic range of Phe concentration varies according to different guidelines [8,9], and there is no international consensus. The US diagnostic and management guidelines recommend that the initiation of treatment for PKU should be undertaken as early as possible, preferably within the first week after birth, with a goal of having blood Phe in the range 120-360 $\mu \mathrm{mol} / \mathrm{L}$ within the first 2 weeks of life, to prevent permanent neurological damage [10]. The European guidelines recommend target concentrations of 120-360 $\mu \mathrm{mol} / \mathrm{L}$ for individuals aged 0-12 years and for maternal PKU [11]. In both, this is largely achieved by a natural proteinrestricted diet and Phe-free synthetic amino-acid supplementation $[10,11]$. However, adherence to a Phe-restricted diet is burdensome owing to the need for long-term dietary counselling and daily micronutrient supplementation [12]. The management guidelines also stipulate that a course of treatment with $\mathrm{BH}_{4}$ should be investigated $[10,11]$.

Sapropterin dihydrochloride (sapropterin, Kuvan ${ }^{\circledR}$, Merck, Geneva, Switzerland, an affiliate of Merck KGaA, Darmstadt, Germany, and BioMarin, Novato, CA, USA) is a synthetic formulation of $\mathrm{BH}_{4}$ that has been shown to be effective in lowering serum Phe concentrations and/or improving dietary Phe tolerance in a subset of patients with PKU or mild HPA who respond to treatment with $\mathrm{BH}_{4}$ (known as responders) and in the rare patients with a defect in $\mathrm{BH}_{4}$ synthesis [12]. Based on the results of the SPARK (Safety Paediatric efficAcy phaRmacokinetics with Kuvan $^{\oplus}$ ) study, the European Medicines Agency has recently extended the indication for sapropterin from the treatment of $\mathrm{BH}_{4}$-responsive $\mathrm{PKU}$ in adults and children aged $\geq 4$ years and in all $\mathrm{BH}_{4}$-deficient adults and children $[12,13]$ to now include children with $\mathrm{BH}_{4}$-responsive $\mathrm{PKU}<4$ years old, for whom the previous standard of care was a Phe-restricted diet.

The primary aim of the SPARK study was to evaluate the efficacy (increase in Phe tolerance, defined as the amount of Phe a patient may consume while maintaining blood Phe concentrations within the target range of 120-360 $\mu \mathrm{mol} / \mathrm{L})$; safety of 26 weeks of treatment with sapropterin dihydrochloride plus a Phe-restricted diet compared with a Phe-restricted diet alone in children $<4$ years of age with $\mathrm{BH}_{4}$-responsive PKU or mild HPA; to document the relationship between exposure and response; and to support the posology in this age-group. Although population pharmacokinetic (PopPK) data for sapropterin have been published for infants and young children in the USA and Canada [14], there are no PopPK data for sapropterin in this age range in the European Union (EU); therefore, a secondary aim of SPARK was to develop a PopPK model for sapropterin in this population. The other secondary endpoints were to document the concentrations of blood Phe during the study and extension periods, to document the change in dietary Phe tolerance, and to monitor blood pressure, growth parameters, and neuromotor developmental milestones.

\section{Methods \\ Study design}

The SPARK trial (NCT01376908) is a 26-week openlabel, multicentre, randomized phase IIIb study to assess the efficacy, safety and PopPK of sapropterin in patients aged $<4$ years with $\mathrm{BH}_{4}$-responsive PKU or mild HPA. SPARK was conducted at 22 sites in nine countries: Austria $(n=2)$, Belgium $(n=2)$, Czech Republic $(n=1)$, Germany $(n=4)$, Italy $(n=5)$, Netherlands $(n=2)$, Slovakia $(n=3)$, Turkey $(n=1)$ and the United Kingdom $(n=2)$. The study was performed in accordance with the protocol and subsequent protocol amendments and with the ethical principles laid down in the Declaration of Helsinki, in accordance with the International Conference on Harmonisation (ICH), Note for Guidance on Good Clinical Practice (ICH Topic E6, 1996) and applicable regulatory requirements. The local ethics committee/institutional review board at each of the participating centres approved the protocol.

\section{Patients}

Male or female patients aged $<4$ years at randomization were eligible for entry into the study if they had participated in the screening protocol $<42$ days before study day 1 , had a confirmed diagnosis of mild HPA or PKU (a defined level of Phe tolerance consistent with a diagnosis of $\mathrm{PKU}, \geq 2$ previous blood Phe concentrations $\geq 400 \mu \mathrm{mol} / \mathrm{L}$ obtained 
on two separate occasions), were responsive to $\mathrm{BH}_{4}$ (a decrease of $>30 \%$ in Phe concentrations following a $20 \mathrm{mg} / \mathrm{kg}$ $\mathrm{BH}_{4}$ challenge of at least $24 \mathrm{~h}$ ), good adherence to dietary treatment and maintenance of blood Phe concentrations within the therapeutic target range $(120-360 \mu \mathrm{mol} / \mathrm{L})$ for 4 months prior to screening or at least the last four values of Phe (either from venous blood or dry blood spot) were to be assessed, out of which $75 \%$ had to be within the above therapeutic range. Patients were excluded if they had used sapropterin or any preparation of $\mathrm{BH}_{4}$ within the previous 30 days (unless for the purposes of the $\mathrm{BH}_{4}$ responsiveness test), had known hypersensitivity to sapropterin, its excipients, or to other approved or non-approved formulations of $\mathrm{BH}_{4}$, or had a previous diagnosis of $\mathrm{BH}_{4}$ deficiency.

Patients' parent(s)/guardian(s) gave written informed consent for participation in the study before any trialrelated procedures were performed. Parent(s) and/or guardian(s) had to be willing to comply with all study procedures, maintain strict adherence to the diet, and be willing and able to provide written, signed informed consent after the nature of the study had been explained and prior to any study procedures. Where required, separate informed consent was obtained from the patients' parents or guardians to obtain samples for pharmacokinetic analysis.

\section{Randomization}

On study day one, patients were randomly assigned 1:1 to $10 \mathrm{mg} / \mathrm{kg} /$ day oral sapropterin dissolved in water to be taken with breakfast (after 4 weeks, sapropterin could be increased to $20 \mathrm{mg} / \mathrm{kg} /$ day if Phe tolerance had not increased by $>20 \%$ vs. baseline) plus a Phe-restricted diet or only a Phe-restricted diet for 26 weeks. After study completion, patients were eligible to enrol in a 3-year extension period (to be reported separately), during which all patients received sapropterin plus a Pherestricted diet (Fig. 1).

\section{Efficacy assessments}

The primary outcome was an improvement in dietary Phe tolerance, defined as the daily amount of Phe (mg/

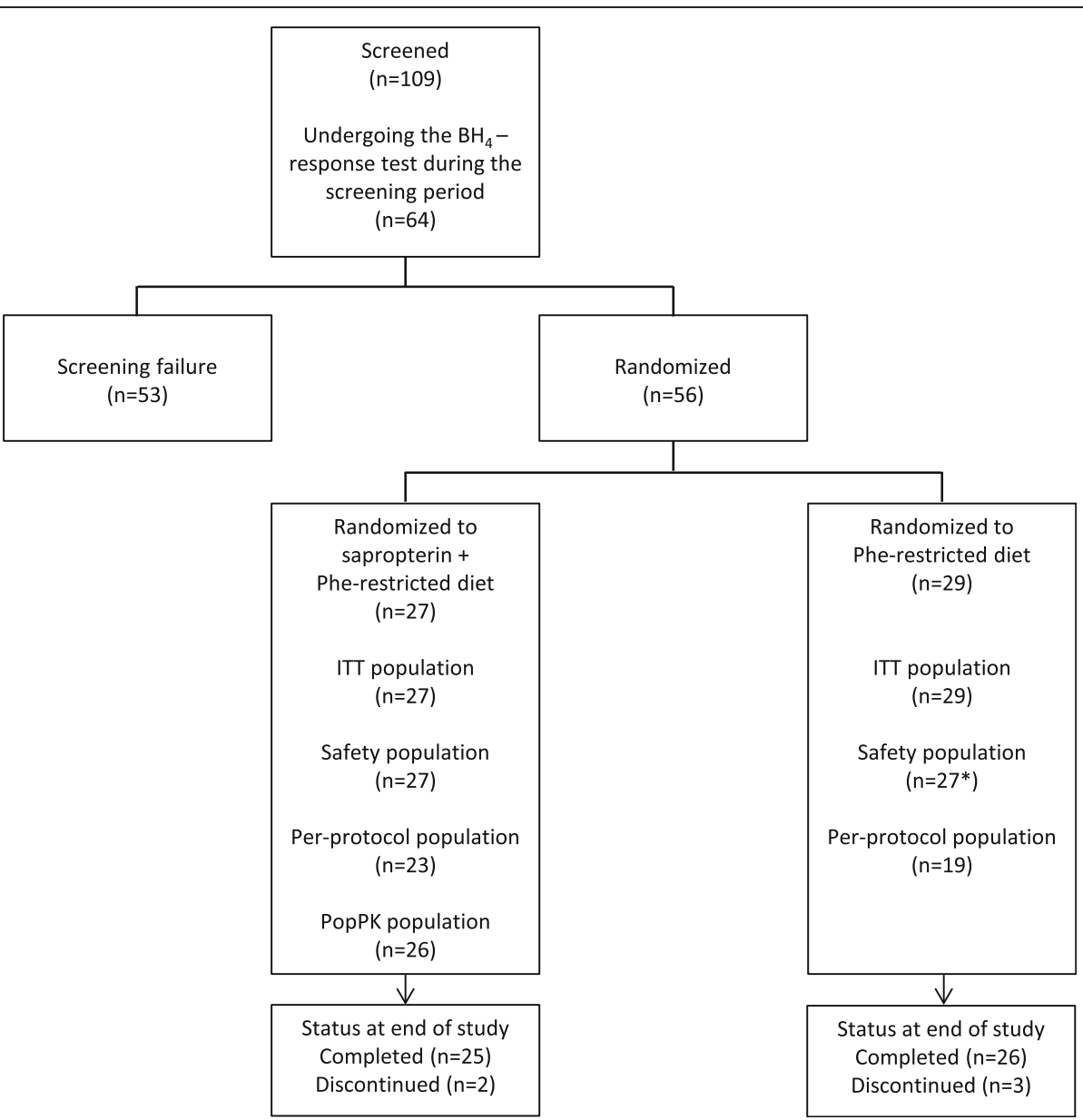

Fig. 1 Patient disposition. *Two of the randomized patients withdrew consent after randomization. No safety assessments were performed during the study period 
Table 1 Algorithm for phenylalanine (Phe) intake adjustments according to mean Phe concentrations

\begin{tabular}{|c|c|}
\hline $\begin{array}{l}\text { Mean Phe } \\
\text { concentration, } \mu \mathrm{mol} / \mathrm{L}\end{array}$ & Phe intake adjustment \\
\hline \multicolumn{2}{|l|}{ At Week 2} \\
\hline $0-300$ & Increase by 5 mg/kg/day \\
\hline $301-359$ & No adjustment required \\
\hline $360-\infty$ & $\begin{array}{l}\text { No adjustment required, but monitor } \\
\text { concentration at next visit }\end{array}$ \\
\hline \multicolumn{2}{|l|}{ Post-Week 2} \\
\hline $0-180$ & Increase by 15 mg/kg/day \\
\hline $181-240$ & Increase by 10 mg/kg/day \\
\hline $241-300$ & Increase by 5 mg/kg/day \\
\hline $301-359$ & No change in dietary Phe intake \\
\hline $360-\infty$ & $\begin{array}{l}\text { Determine if the subject had one or } \\
\text { more previous dietary Phe intake increases } \\
\text { - If so, remove the dietary Phe in the order } \\
\text { that it was previously increased, beginning } \\
\text { with the amount of the last increase } \\
\text { - If not, no adjustment required }\end{array}$ \\
\hline $360-1199$ & $\begin{array}{l}\text { If this is first occasion at this concentration, } \\
\text { monitor concentration at the next visit and } \\
\text { if second occasion is at this concentration, } \\
\text { provide dietary counseling }\end{array}$ \\
\hline $1200-\infty$ & $\begin{array}{l}\text { If this is first occasion at this concentration, } \\
\text { provide dietary counseling and monitor } \\
\text { concentration at the next visit and if second } \\
\text { occasion is at this concentration, provide } \\
\text { dietary counseling and terminate from the trial }\end{array}$ \\
\hline
\end{tabular}

$\mathrm{kg} /$ day) that could be ingested while sustaining mean blood Phe concentrations within a target range of 120$360 \mu \mathrm{mol} / \mathrm{L}$ by dietary Phe adjustments following an algorithm (Table 1). An additional supportive analysis was performed, in which dietary Phe tolerance was based on the Phe intake reported in a 3-day Phe diet diary used to monitor the adherence to the Phe-restricted diet. Analysis and adjustment of dietary intake were performed by the investigator and/or experienced dietician/nutritionist every 2 weeks during the study, according to the study algorithm.

Blood Phe concentrations were measured twice weekly via dried blood spot cards using a high-performance liquid chromatography/tandem mass spectrometry method for Phe detection. The results were verified every 3 months using venous blood plasma. Blood Phe samples could be obtained more frequently at the investigator's discretion.

Secondary endpoints included neuromotor development and physical growth parameters (height or length, weight and maximal occipital-frontal head circumference). Neuropsychological development was assessed using the adaptive behaviour composite score with the Bayley III and the social-emotional composite score in the WPPSI-III, although these results are not reported in this manuscript.

\section{Pharmacokinetic analysis}

The PopPK analysis population comprised all randomized subjects with $\geq 1$ pharmacokinetic sample. PopPK parameters were apparent clearance (CL/F), apparent volume of distribution $(\mathrm{V} / \mathrm{F})$, absorption rate constant $\left(\mathrm{K}_{\mathrm{a}}\right)$, and endogenous $\mathrm{BH}_{4}(\mathrm{C} 0)$. These were used to compute the area under the curve $\left(\mathrm{AUC}_{0-\infty}\right)$, peak serum concentration $\left(\mathrm{C}_{\max }\right)$, time of $\mathrm{C}_{\max }\left(\mathrm{T}_{\max }\right)$, and half-life $\left(\mathrm{t}_{1 / 2}\right)$. Plasma samples were collected for endogenous $\mathrm{BH}_{4}$ measurement at baseline and sparsely thereafter between weeks 5-12 after oral administration of sapropterin $10 \mathrm{mg} / \mathrm{kg} /$ day. In order to ensure that the sparse pharmacokinetic sampling provided sufficient information and that samples were taken at informative times, the sampling had been planned using D-optimization [15]. During this process, competing maturation functions were considered $[16,17]$.

PopPK modelling was conducted using NONMEM $^{\circ}$ (software version 7, level 2; Icon Development Solutions, Ellicott City, MD, USA) using standard model building and evaluation approaches. Covariates, including age, weight and sex, were evaluated using standard methodology to determine if these factors were predictive of $\mathrm{BH}_{4}$ pharmacokinetics. The final model was subsequently used to derive metrics of exposure and to determine the exposure relative to adult PKU patients.

\section{Laboratory assessments}

All standard blood chemistry, hematologic and urine analysis, as well as specialized testing for Phe and tyrosine concentrations, were performed at a central laboratory.

\section{Safety analysis}

The safety population consisted of all subjects who had some safety assessment data available. Safety was assessed at the clinic on a monthly basis during the 26-week study period or until 4 weeks post-treatment, by recording, reporting, and analysis of baseline medical conditions and adverse events (AEs) and physical examination findings (including vital signs). Standard blood chemistry, hematologic, and urine analyses were performed every 3 months during the study period for safety analysis.

\section{Genotype analysis}

$P A H$ genotype data were collected at screening for enrolled patients, after a separate informed consent was obtained from the patients' parents or guardians. Genotype testing was performed by a central laboratory.

\section{Statistical analyses}

The primary efficacy analysis population was the intention-to-treat (ITT) population comprising all randomized patients. The per-protocol (PP) population included all ITT patients who completed the study with 
no prohibited concomitant medication and without major protocol deviation. A missing pre-study Phe tolerance, lack of adherence to Phe-restricted diet over the past 3 months, lack of adherence to sapropterin, and a sapropterin-dose adjustment not conducted as per protocol were considered to be major protocol deviations leading to exclusion from the PP population. The safety population comprised all patients with safety assessment data available ( $\geq 1$ visit for vital signs, AEs or laboratory results) and who had received $\geq 1$ dose of sapropterin or were randomly assigned to Phe-restricted diet alone.

The sample size was planned to be 23 patients per group, to ensure a power of $80 \%$ to demonstrate a treatment group difference, assuming a dietary Phe tolerance of $20 \mathrm{mg} / \mathrm{kg} /$ day under dietary therapy alone, a difference of $75 \%$ with the sapropterin plus diet group, and a common standard deviation of $17.5 \mathrm{mg} / \mathrm{kg} /$ day. To compensate for possible dropouts, a total of 50 subjects were to be randomized.

The dietary Phe tolerance was analyzed using the repeated measures analysis of covariance (ANCOVA) on the observed records for the ITT population, with baseline Phe tolerance, treatment group, age group, visit, baseline blood Phe concentration and treatment by visit interaction as fixed effects. Secondary endpoints were described using summary statistics.

Non-linear mixed-effect modelling (NONMEM ${ }^{\circ}$ software version 7, level 2) was applied to estimate the pharmacokinetic parameters and their variability. The final model was evaluated using a number of methods, which included bootstrapping and visual predictive checks, as conducted previously in children aged 0-6 years [18].

In order to evaluate the differences in exposure expected from the original model and the current model, simulated concentration-time profiles for the reference subject were generated.

\section{Results}

\section{Patient disposition and demographics}

In total, 109 patients were screened (Table 2 and Fig. 1), of whom 53 were screening failures (49 patients did not meet eligibility criteria and four patients for other reasons). Fifty-six patients were randomized (27 patients to the sapropterin plus Phe-restricted diet group and 29 patients to the diet-only group). Fifty-two patients were included in the PopPK population. Patients were stratified according to age: 15 patients were $<12$ months, 18 patients were 12 to $<24$ months, and 23 patients were 24 to $<48$ months. A numerically higher proportion of patients in the sapropterin plus Phe-restricted diet group successfully followed the protocol compared with patients in the Phe-restricted diet only group $(85 \%$ [23 of 27 patients] vs. 65\% [19 of 29 patients]). The
Table 2 Demographic and baseline characteristics (ITT population)

\begin{tabular}{|c|c|c|}
\hline Characteristic & $\begin{array}{l}\text { Sapropterin + Phe- } \\
\text { restricted diet }(n=27)\end{array}$ & $\begin{array}{l}\text { Phe-restricted } \\
\text { diet only }(n=29)\end{array}$ \\
\hline \multicolumn{3}{|l|}{ Age, months } \\
\hline mean士SD & $21.1 \pm 12.3$ & $21.2 \pm 12.0$ \\
\hline $\min ; \max$ & $2 ; 47$ & $2 ; 44$ \\
\hline \multicolumn{3}{|l|}{ Age group, n (\%) } \\
\hline$<12$ months & $7(25.9)$ & $8(27.6)$ \\
\hline $12-<24$ months & 9 (33.3) & $9(31.0)$ \\
\hline $24-<48$ months & $11(40.7)$ & $12(41.4)$ \\
\hline Males, n (\%) & $16(59.3)$ & $14(48.3)$ \\
\hline \multicolumn{3}{|l|}{ Height, cm } \\
\hline mean $\pm S D$ & $82.0 \pm 11.3$ & $82.3 \pm 11.6$ \\
\hline $\min ; \max$ & $59 ; 108$ & $57 ; 105$ \\
\hline \multicolumn{3}{|l|}{ Weight, kg } \\
\hline mean $\pm S D$ & $11.3 \pm 3.1$ & $11.3 \pm 2.8$ \\
\hline $\min ; \max$ & $5 ; 20$ & $6 ; 16$ \\
\hline \multicolumn{3}{|l|}{ BMI, kg/m² } \\
\hline meantSD & $16.5 \pm 1.0$ & $16.5 \pm 1.4$ \\
\hline $\min ; \max$ & $14 ; 18$ & $14 ; 20$ \\
\hline \multicolumn{3}{|c|}{ Age at PKU diagnosis, days } \\
\hline mean $\pm S D$ & $27.2 \pm 79.8$ & $32.6 \pm 72.2$ \\
\hline $\min ; \max$ & $1 ; 425$ & $4 ; 382$ \\
\hline \multicolumn{3}{|c|}{ Blood Phe concentration at diagnosis, $\mu \mathrm{mol} / \mathrm{L}$} \\
\hline mean+SD & $780.3 \pm 480.7$ & $879.9 \pm 596.5$ \\
\hline $\min ; \max$ & $191 ; 2062$ & $221 ; 2600$ \\
\hline \multicolumn{3}{|l|}{ PKU severity ${ }^{a} \mathrm{n}(\%)$} \\
\hline Classical PKU & $5(18.5)$ & $7(24.1)$ \\
\hline Mild PKU & $10(37.0)$ & $8(27.6)$ \\
\hline Mild HPA & $12(44.4)$ & $14(48.3)$ \\
\hline
\end{tabular}

$B M I$ body mass index, HPA hyperphenylalaninemia, ITT intention-to-treat, $P h e$ phenylalanine, $P K U$ phenylketonuria, SD standard deviation

${ }^{\mathrm{a}}$ Disease severity according to blood Phe concentrations: Classical PKU,

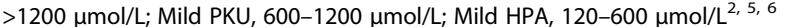

mean ( \pm standard deviation $[\mathrm{SD}])$ age at diagnosis was $30( \pm 75.3)$ days. Almost half $(46.4 \%)$ were diagnosed with mild HPA, 32.1\% were diagnosed with mild PKU, and $21.4 \%$ were diagnosed with classical PKU.

The overall mean adherence to sapropterin (defined as the proportion between the actual dose administered and the prescribed dose) over the study was 100\% (range 82 to $107 \%)$. Most patients $(n=25,92.6 \%)$ continued on $10 \mathrm{mg} / \mathrm{kg} /$ day after 4 weeks of treatment, with only two patients switching to $20 \mathrm{mg} / \mathrm{kg} /$ day. The overall mean $( \pm \mathrm{SD})$ adherence to diet, as assessed by a 3-day food diary, was $94.6 \pm 9.4 \%$ (range 69 to $111 \%$ ) in the sapropterin-treated group and $92.1 \pm 23.8 \%$ (range 65 to $183 \%)$ in the diet-only treated group. 


\section{Dietary Phe tolerance after 26 weeks}

At week 26, the adjusted mean dietary Phe tolerance was higher in the sapropterin plus Phe-restricted diet group compared with the diet-only group. The tolerance based on prescribed Phe was $80.6 \mathrm{mg} / \mathrm{kg} /$ day vs. $50.1 \mathrm{mg} / \mathrm{kg} /$ day (adjusted between-group difference $30.5 \mathrm{mg} / \mathrm{kg} /$ day [95\% confidence interval (CI) 18.7, 42.3], $p<0.001)$. The tolerance based on reported dietary Phe tolerance from the intake diary was $75.7 \mathrm{mg} / \mathrm{kg} /$ day [ $95 \%$ CI $67.2,84.11$ ] vs. $42.0 \mathrm{mg} / \mathrm{kg} /$ day [95\% CI 33.1, 50.8] (adjusted between-group difference 33.7 [95\% CI 21.4, 45.9], $p<0.001$; Fig. 2a). A similar difference was reported in the per-protocol population (adjusted between-group difference 36.4 [95\% CI 25.4, 47.4], $p<0.001)$. In addition, consistent results were seen in the ITT population following supportive analysis on diary-recorded Phe intake.

\section{Blood Phe concentrations}

Phe concentrations from dried blood spots were lower than those from venous blood spots but this was consistent with differences reported in the literature [19-21]. In the Phe-restricted diet group, the adjusted mean blood Phe concentrations in the ITT population were stable over time, with a mean $( \pm \mathrm{SD})$ increase of 23.1 $( \pm 21.9) \mu \mathrm{mol} / \mathrm{L}$ at week 26 (Fig. 2b). In the sapropterin plus Phe-restricted diet group, the mean $( \pm S D)$ blood concentrations decreased by $110.7( \pm 20.1) \mu \mathrm{mol} / \mathrm{L}$ at week 4 and gradually returned to concentrations similar to those seen in the Phe-restricted diet group, reflecting the increase in Phe intake and Phe tolerance. At week 26, the adjusted mean $( \pm \mathrm{SD})$ blood Phe concentrations were similar: $300.1( \pm 115.2) \mu \mathrm{mol} / \mathrm{L}$ in the sapropterin plus Phe-restricted diet group and $343.3( \pm 118.4) \mu \mathrm{mol} / \mathrm{L}$ in the diet-only group (adjusted between-group difference $33.2 \mu \mathrm{mol} / \mathrm{L}[95 \% \mathrm{CI}-94.8,28.4], p=0.290)$. It is important to note that patients were expected to maintain blood Phe concentrations within this range; therefore, differences in blood Phe concentrations were not anticipated.

The observed proportion of patients with blood Phe concentrations maintained in the range 120-360 $\mu \mathrm{mol} / \mathrm{L}$ throughout the whole study was greater in the sapropterin plus Phe-restricted diet group $(n=9 / 27,33.3 \%)$ than in the diet-only group $(n=3 / 29,10.3 \%) .21$ of 27 (77.8\%) sapropterin-treated patients and 15 of 27 (55.6\%) patients on only the Phe-restricted diet had $\geq 1$ blood Phe concentration at or below the $120 \mu \mathrm{mol} / \mathrm{L}$ threshold established by the British PKU Registry [22]. However, very few instances of Phe concentration below the normal range thresholds of 40 and $26 \mu \mathrm{mol} / \mathrm{L}$ were observed during the study.
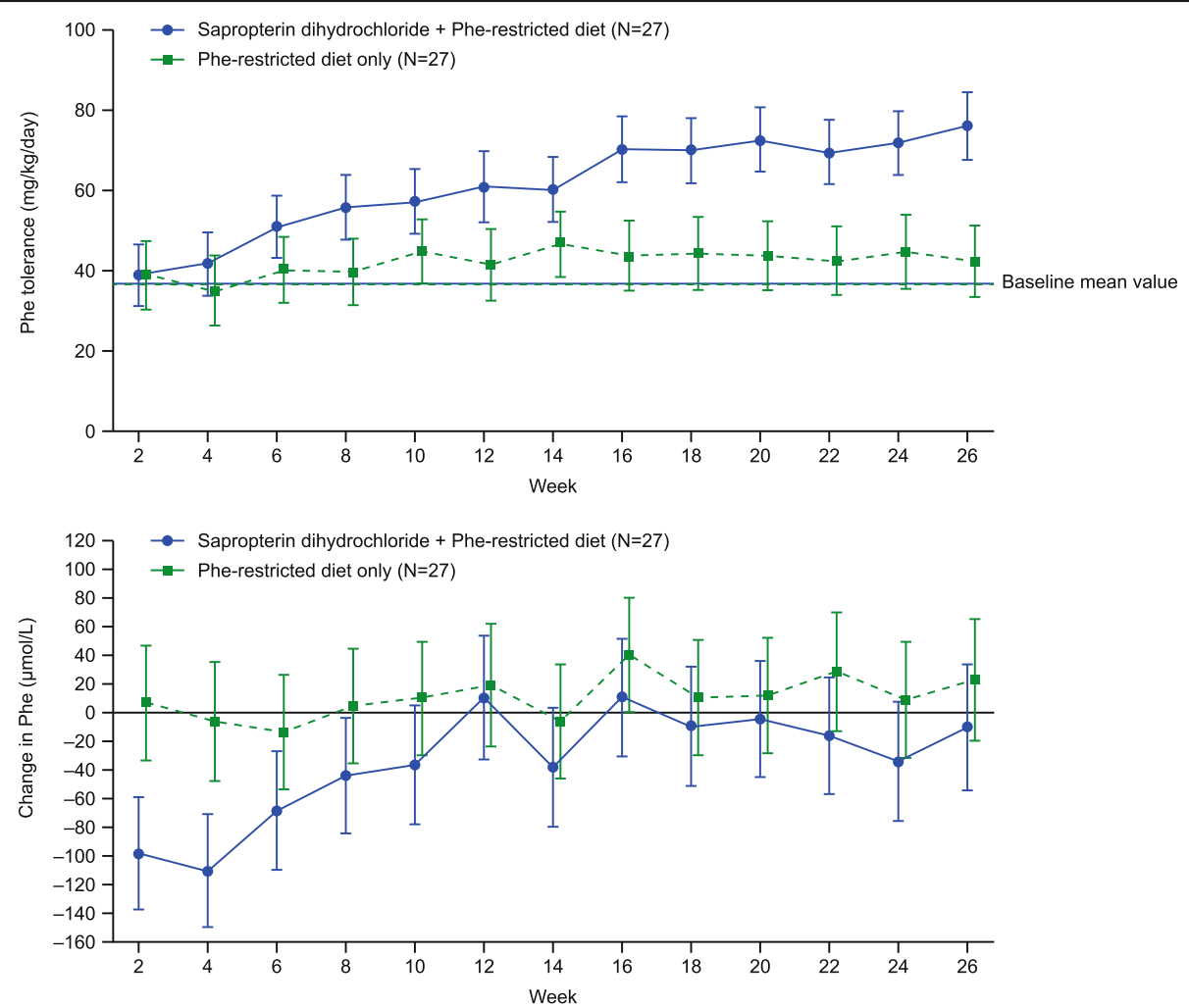

Fig. 2 a Adjusted mean dietary Phe tolerance ( $\mathrm{mg} / \mathrm{kg} / \mathrm{day})$ a and mean Phe change from baseline ( $\mu \mathrm{mol} / \mathrm{L}) \mathbf{b}$ Error bars represent $95 \%$ confidence intervals. Phe, phenylalanine. Cl, confidence interval; Phe, phenylalanine 


\section{Change from baseline in dietary Phe tolerance}

The mean change in dietary Phe tolerance between baseline and the last Phe tolerance observation was assessed within each treatment group. The mean $( \pm \mathrm{SD})$ change from baseline to week 26 in patients receiving sapropterin plus Phe-restricted diet was $36.9( \pm 27.3) \mathrm{mg} / \mathrm{kg} /$ day $(p<0.001)$. The mean change from baseline in patients only on the Phe-restricted diet was $13.1( \pm 19.6) \mathrm{mg} / \mathrm{kg} /$ day $(p=0.002)$.

\section{Pharmacokinetic analysis}

The pharmacokinetic data are best described by a onecomponent model with first-order input following a time lag and first-order elimination, with an endogenous baseline $\mathrm{BH}_{4}$ concentration component. The model included terms describing between-subject variability on apparent clearance $(\mathrm{CL} / \mathrm{F})$ and apparent volume of distribution $(\mathrm{V} / \mathrm{F})$ as well as their correlation (Table 3). The final model parameter estimate for $\mathrm{CL} / \mathrm{F}$ was $2780 \mathrm{~L} / \mathrm{h}$, $3870 \mathrm{~L}$ for $\mathrm{V} / \mathrm{F}$, and $0.234 \mathrm{~h}^{-1}$ for $\mathrm{K}_{\mathrm{a}}$.

From the model, an elimination half-life of approximately $1 \mathrm{~h}$ can be computed, with an absorption half-life $\left(\ln 2 / \mathrm{K}_{\mathrm{a}}\right)$ of approximately $3 \mathrm{~h}$, suggesting flip-flop kinetics where the absorption becomes the rate-limiting step of drug disposition.

Body weight was the only covariate that affected the $\mathrm{CL} / \mathrm{F}$ and V/F of sapropterin: these variables increased in a nonlinear manner with increasing weight, although individual predictions still varied around the typical individual predictions (Fig. 3). At the lowest extreme of weight, a $5 \mathrm{~kg}$ patient had a CL/F value $11 \%$ of that of a $70 \mathrm{~kg}$ reference adult, and a V/F value $22 \%$ of that of the reference adult (Table 4).

Even after inclusion of weight into the pharmacokinetic model, significant between-subject variability in

Table 3 Parameter estimates for final model

\begin{tabular}{lll}
\hline & Population mean & SE\% \\
\hline CL/F (L/h) & 2780 & 2.0 \\
V/F $(L)$ & 3870 & 5.9 \\
$K_{a}(1 / h)$ & 0.234 & 6.6 \\
LAG (h) & 0.342 & 2.8 \\
CO ( $\mu$ g/L) & 12.6 & 7.8 \\
Coefficient describing effect of weight on CL/F & 0.839 & 1.8 \\
Coefficient describing effect of weight on V/F & 0.573 & 3.3 \\
Residual error (\%CV) & 65.30 & 8.5 \\
IIV_CL (\%CV) & 22.98 & 0.2 \\
IIV_V2 (\%CV) & 32.56 & 0.2 \\
Corr (CL,V) & 0.134 & $\mathrm{NE}$
\end{tabular}

SE standard error, $C L / F$ apparent clearance, $V / F$ apparent volume of distribution, $L A G$, lag time, $K_{a}$ absorption rate constant, $C O$ endogenous $\mathrm{BH}_{4}$ concentrations, $C V$ coefficient of variation, IIV between-subject variability, $N E$ not estimated

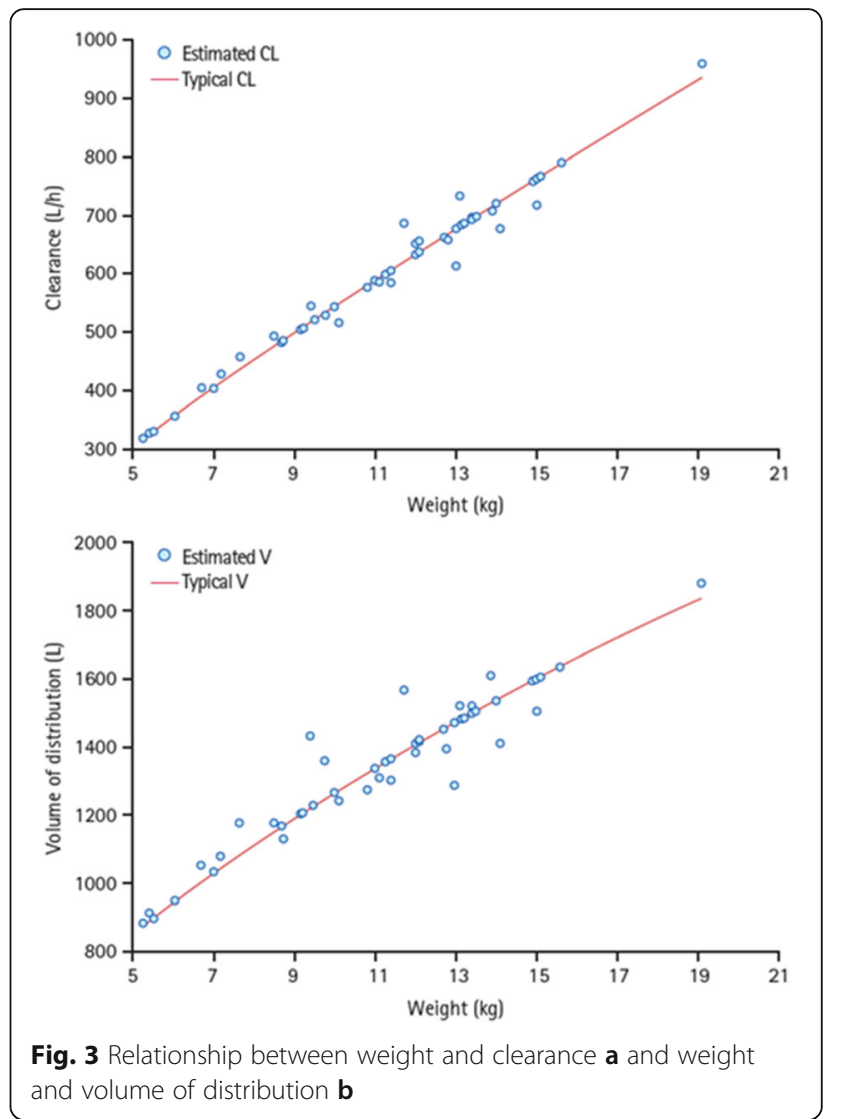

$\mathrm{CL} / \mathrm{F}$ and V/F remained, supporting an adaptive approach to individual treatment. Simulated concentration-time curves following sapropterin $10 \mathrm{mg} / \mathrm{kg}$ show that sapropterin concentrations remain above the modelestimated endogenous $\mathrm{BH}_{4}$ concentrations $(12.6 \mu \mathrm{g} / \mathrm{L}$; Table 3) for the dose interval for patients with different weights (Fig. 4).

Overall, the exposure across all age groups is comparable, although the number of patients in all age groups is small. The exposure in pediatric patients was lower than the expected exposure in adults, based on the simulated concentration-time profiles following the $10 \mathrm{mg} / \mathrm{kg} /$ day dose across a range of body weights. This analysis shows that the concentrations remain above the endogenous concentration, which is set at a concentration

Table 4 Effect of weight on clearance and volume of distribution

\begin{tabular}{llclc}
\hline Weight $(\mathrm{kg})$ & $\mathrm{CL} / \mathrm{F}(\mathrm{L} / \mathrm{h})$ & \% of reference & $\mathrm{V} / \mathrm{F}(\mathrm{L})$ & \% of reference \\
\hline 5 & 305 & 10.9 & 853 & 22.0 \\
15 & 766 & 27.5 & 1601 & 41.4 \\
25 & 1176 & 42.2 & 2145 & 55.4 \\
$70^{\mathrm{a}}$ & 2789 & 100.0 & 3870 & 100.0
\end{tabular}

CL/F apparent clearance, $V / F$ apparent volume of distribution ${ }^{\mathrm{a}}$ Reference weight (adult male patient) 


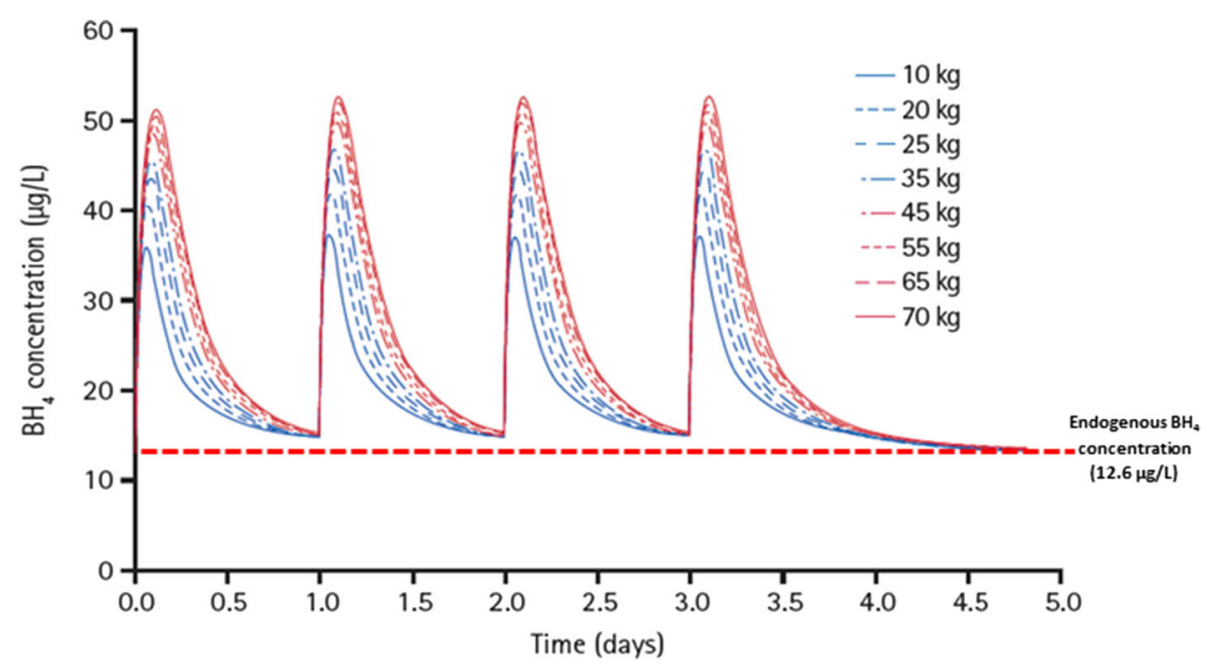

Fig. 4 Simulated concentration-time curves for patients with various weights following sapropterin (10 mg/kg/day)

below that for a person not diagnosed with PKU, for a daily dose interval and support the current approach to treatment as conservative (Fig. 4).

\section{Safety}

The safety population comprised 54 patients; two of the randomized patients withdrew consent after randomization and, therefore, were excluded from the safety population (Fig. 1). All patients in the safety population reported at least one $\mathrm{AE}$ (Table 5); in the sapropterin plus Pherestricted diet group, eight out of 27 patients $(29.6 \%)$ reported at least one treatment-emergent AE (TEAE) classified as related to sapropterin. The proportion of patients reporting TEAEs was the same in the two groups, and no

Table 5 Summary of safety data showing the proportion of patients reporting adverse events (AEs) (Safety population)

\begin{tabular}{|c|c|c|c|c|}
\hline & \multicolumn{2}{|c|}{ Sapropterin + Phe-restricted diet $(n=27)$} & \multicolumn{2}{|c|}{ Phe-restricted diet alone $(n=27)$} \\
\hline & Patients, n (\%) & Events, $\mathrm{n}$ & Patients, n (\%) & Events, $\mathrm{n}$ \\
\hline Treatment-emergent AEs & $27(100)$ & 282 & $27(100)$ & 278 \\
\hline AEs related to sapropterin & $8(29.6)$ & 31 & NA & NA \\
\hline Infections and infestations related to sapropterin & $3(11.1)$ & 3 & NA & NA \\
\hline Gastrointestinal disorders related to sapropterin & $3(11.1)$ & 8 & NA & NA \\
\hline Amino acid concentrations decrease related to sapropterin & $6(22.2)$ & 20 & NA & NA \\
\hline SAES & $3(11.1)$ & 5 & $1(3.7)$ & 2 \\
\hline Gastroenteritis & $1(3.7)$ & 1 & $0(0.0)$ & 0 \\
\hline Rash & $1(3.7)$ & 1 & $0(0.0)$ & 0 \\
\hline Overdose $^{a}$ & $1(3.7)$ & 2 & $0(0.0)$ & 0 \\
\hline Stomatitis & $1(3.7)$ & 1 & $0(0.0)$ & 0 \\
\hline Bronchiolitis & $0(0.0)$ & 0 & $1(3.7)$ & 1 \\
\hline Bronchopneumonia & $0(0.0)$ & 0 & $1(3.7)$ & 1 \\
\hline AEs leading to discontinuation & $0(0.0)$ & 0 & $0(0.0)$ & 0 \\
\hline
\end{tabular}

NA not appropriate, $S A E$ serious $A E$

${ }^{a}$ On the day of first administration of study treatment, the subject had a sapropterin overdose (severity: mild; $80 \mathrm{mg} / \mathrm{day}$ instead of $75 \mathrm{mg} / \mathrm{day}$ by mistake). At 26 days after the first administration of study treatment, the subject had another sapropterin overdose (severity: $\mathrm{mild} ; 80 \mathrm{mg} / \mathrm{day}$ instead of $75 \mathrm{mg} / \mathrm{day}$ by mistake). Both events were reported in accordance with the protocol and were therefore categorized as medically important. The subject recovered without sequelae from both events. The administration of sapropterin plus Phe-restricted diet alone was continued without change after the first overdose and the dose was reduced after the second overdose

AE defined as any untoward medical occurrence in a subject or clinical investigation subject administered a pharmaceutical product, which did not necessarily have a causal relationship with trial treatment; SAE was any untoward medical occurrence that at any dose: resulted in death; was life-threatening; might have caused death if it had been more severe; required inpatient hospitalization or prolongation of existing hospitalization; resulted in persistent or significant disability/incapacity; was a congenital anomaly/birth defect; or was otherwise considered as medically important 
patients withdrew owing to AEs. None of the TEAEs were graded as severe. All patients had at least one TEAE that was judged to be mild in severity. Seven (25.9\%) patients in the sapropterin plus Phe-restricted diet group had nine TEAEs graded as moderate in severity, and eight (29.6\%) patients in the Phe-restricted diet group reported 18 TEAEs graded as moderate in severity.

The most common TEAEs in the sapropterin plus Phe-restricted diet group and in the Phe-restricted diet group were: pyrexia (63.0 and 66.7\%), cough (48.1 and $48.1 \%$ ) and nasopharyngitis (48.1 and $40.7 \%$ ), respectively. The most common TEAEs classified as related to sapropterin were amino acid concentration decrease (six patients [22.2\%]), rhinitis, and vomiting (two patients each [7.4\%]), and one patient $(3.7 \%)$ each for pharyngitis, diarrhea, abdominal pain, mouth ulceration and increased amino acid concentration.

Although the proportion of patients who reported a serious AE (SAE) was higher in the sapropterin plus Phe-restricted diet group compared with the Phe-restricted diet (11.1 vs. 3.7\%), all SAEs were assessed as unrelated to sapropterin treatment (Table 5).

\section{Genotype data}

Of 109 patients who were screened, 73 agreed to participate in the pharmacogenetics sub-study. Of the 73 patients who agreed, 36 were screening failures, leaving genotype data for 37 responders (Additional file 1: Table S1).

\section{Neuromotor development and growth parameters}

Most patients in both treatment groups had normal neuromotor development, including fine motor, gross motor, language, and personal and social function, and there were no statistically significant differences between treatment groups in any of the neuromotor developmental milestones at baseline, 12 and 26 weeks (Additional file 1: Figure S1).

Patients in both treatment groups had stable growth parameters, including body mass index SD score (SDS), height SDS, maximum occipital-frontal head circumference SDS and weight SDS. There were no statistically significant differences between the treatment groups for any of the growth parameters.

\section{Discussion}

In PKU, blood Phe concentrations need to be controlled from birth to prevent neurological sequelae, such as cognitive impairment and mild-to-severe intellectual disability, linked to PKU [5, 7]. Until July 2015 there was no licensed pharmacological treatment available in the EU for children with PKU aged $<4$ years, and the standard of care was a Phe-restricted diet. The results of the SPARK study, which was the first clinical trial of sapropterin in patients 0-4-years-old with $\mathrm{BH}_{4}$-responsive PKU or mild HPA in Europe, showed that daily dosing with 10 or $20 \mathrm{mg} / \mathrm{kg} /$ day sapropterin in combination with a Phe-restricted diet led to statistically and clinically significant improved dietary Phe tolerance at week 26 compared with a Phe-restricted diet alone, while maintaining mean blood Phe concentrations within the protocol-specified range. These results were consistent with those seen in children aged 4-12 years treated with $20 \mathrm{mg} / \mathrm{kg} /$ day sapropterin, in whom the mean amount of Phe supplement tolerated had increased at 10 weeks of treatment [23]. The results were also consistent with those reported in a study from the USA and Canada in children aged 0-6 years old, in whom $20 \mathrm{mg} / \mathrm{kg} /$ day sapropterin treatment lowered blood Phe concentrations, enabling, in some cases, an increase in dietary Phe intake [24].

The benefits of initiating sapropterin therapy in patients younger than 4 years have been highlighted by a post-marketing study conducted in Japan between 1995 and 2001, which reported that all patients who started treatment with sapropterin before the age of 4 years maintained serum Phe concentrations within the recommended range for the duration of the study [25]. Previous reports have shown that neurocognitive function was preserved and no neurodevelopmental penalty was reported in patients who started sapropterin therapy between 0 and 6 years of age [24], and that treatment with $\mathrm{BH}_{4}$ may enable relaxation of the dietary regimen, leading to improved quality of life [26]. Patients with mild HPA, who comprised almost a half of the population in this study, retain substantial enzyme activity and will, therefore, likely respond to sapropterin treatment. However, the indication for treatment of mild HPA differs between countries due to weak evidence. US guidelines recommend treatment at a Phe concentration above $360 \mu \mathrm{mol} / \mathrm{L}$ [10], while other countries start treatment at Phe concentrations above $600 \mu \mathrm{mol} / \mathrm{L}$ [27].

In this study, the addition of sapropterin to a Pherestricted diet in patients $<4$ years old with $\mathrm{BH}_{4}$-responsive PAH deficiency significantly improved Phe tolerance compared with a Phe-restricted diet alone. In the sapropterintreated group, blood Phe concentrations initially fell at the beginning of treatment ( 4 weeks), but they slowly increased over the course of the study to reach concentrations similar to those at baseline by week 12 (Fig. 2), while increasing dietary Phe-intake. The observed increase in Phe tolerance reported in patients on the Phe-restricted diet compared with the tolerance at baseline may be explained by the fact that the patients in this group were not at their maximum Phe tolerance in daily practice before starting the study. This observation confirms the expectation that under the tight control of study conditions using a strict algorithm of Phe escalation, dietary Phe tolerance may be further optimized [28]. Because of the potential for Phe concentrations to drop below either the normal or the desired therapeutic concentrations owing to the action of 
sapropterin, careful monitoring and adjustment of therapeutic dose and dietary Phe concentrations was necessary.

The pharmacokinetics of $\mathrm{BH}_{4}$ can be well described by a one-compartment model that respects the principle of parsimony and provides accurate estimates that describe $\mathrm{BH}_{4}$ profiles virtually identical to those from a twocompartment model evaluated in a previous study [18]. The terminal and absorption half-lives are suggestive of flip-flop pharmacokinetic behavior, in which absorption is the rate-limiting step of drug disposition. Sapropterin exposure was similar across all age groups studied here. With this in mind, a once-daily dosing regimen is justified. Weight was the only covariate that had an effect on the clearance and volume distribution of sapropterin, meaning that dose adjustments based on weight are appropriate [14].

The secondary endpoints of growth and neuromotor development were considered to be normal in the patient population throughout the study and no difference between groups was observed, suggesting no treatment effect on these growth and development parameters. However, the time scale in the study was too short to expect clinically meaningful changes in neuromotor development.

The safety profile for sapropterin was acceptable and similar to that reported in studies of patients $>4$ years old [23] and in those $<4$ years old [25], with no deaths, severe TEAEs or withdrawals reported. Although four patients had SAEs, none of these was deemed to be related to treatment. The number of TEAEs was similar between the two groups and was commonly associated with normal childhood illness.

\section{Conclusion}

In conclusion, the addition of sapropterin to a Pherestricted diet in patients aged $<4$ years old with $\mathrm{BH}_{4}$-responsive PKU, mild PKU or mild HPA was well tolerated and led to a significant improvement in Phe tolerance compared with only a Phe-restricted diet. The pharmacokinetics of sapropterin in patients aged $<4$ years are adequately described by a onecompartment model, and favor once-a-day dosing with dose adjustment for weight. These data led to the approval of sapropterin for individuals with $\mathrm{BH}_{4}$ responsive PKU or mild HPA aged $<4$ years, and will thus change treatment management for this subset of patients in the first week of life.

\section{Additional file}

Additional file 1: Figure S1. Summary of neuromotor developmental milestones - ITT population. Data show (a) the proportion of patients with normal development in the area of assessment at baseline, (b) Week
12, and (c) Week 26 treated with sapropterin plus the Phe-restricted diet, or Phe-restricted diet alone. P-values show comparison of results between treatment groups at Week 26 using the chi-squared test. Table S1. PAH genotypes of sapropterin responders $(n=37)$. (DOCX $180 \mathrm{~kb}$ )

\section{Abbreviations}

AE: Adverse event; ANCOVA: Analysis of covariance; AUC: Area under the curve; $\mathrm{CO}$ : Endogenous $\mathrm{BH}_{4} ; \mathrm{C}_{\text {max }}$ : Peak serum concentration; $\mathrm{Cl}$ : Confidence interval; CL/F: Apparent clearance; EU: European Union;

HPA: Hyperphenylalaninemia; ICH: International Conference on Harmonisation; ITT: Intention-to-treat; $\mathrm{K}_{\mathrm{a}}$ : Adsorption rate constant; PAH: Phenylalanine hydroxylase; Phe: Phenylalanine; PKU: Phenylketonuria; PopPK: Population pharmacokinetics; PP: Per-protocol; SAE: Serious AE; SD: Standard deviation; SDS: SD score; SPARK: Safety paediatric efficacy pharmacokinetics with Kuvan ${ }^{\oplus ;} T_{1 / 2}$ : Half-life; $T_{\text {max }}$ : Time of $C_{\text {maxi }}$;

TEAE: Treatment-emergent AE; V/F: Apparent volume of distribution

\section{Acknowledgements}

The authors would like to thank patients, investigators, co-investigators, and the study teams at each of the participating centres and at Merck KGaA, Darmstadt, Germany and EMD Serono, Boston, MA, USA. The authors would also like to thank Anita MacDonald who contributed significantly to the design and running of the study, including suggesting changes to the protocol, dietetic monitoring requirements, and helping to recruit patients. The authors would like to acknowledge the SPARK investigators for their contribution, without whom this study would not be possible: Dr Med Michaela Brunner-Krainz (University Hospital, Graz, Austria); Professor Daniela Karall (Clinic for Pediatrics I, Innsbruck, Austria); Professor Philippe Goyens (Hôpital Universitaire des Enfants, Brussels, Belgium); Dr Patrick Verloo (UZ Gent, Belgium); Dr Giovanna Cotugno (Ospedale Pediatrico Bambino Gesù, Rome, Italy); Dr Enrica Riva (Clinica Pediatrica - Ospedale San Paolo, Milan, Italy); Dr Annet Bosch (Academisch Medisch Centrum, Amsterdam, The Netherlands); Dr Estela Rubio-Gozalbo (Maastricht Universitair Medisch Centrum; Maastricht, The Netherlands); Dr Katarina Hálová (Detská fakultná nemocnica s poliklinikou Banska Bystrica, Slovakia); Dr Ludmila Potocnakova (Detská fakultná nemocnica Kosice, Metabolická ambulancia, Kosice, Slovakia); Dr Oto Urge (Univerzitna nemocnica Bratislava, Nemocnica sv. Cyrila a Metoda, Bratislava, Slovakia); Dr llaria Bettocchi, Graziana D'Addabbo, Carla Musiani (Department of Pediatrics, University of Bologna, Bologna, Italy). Finally, the authors thank Steven Goodrick and Andrew Thomson of inScience Communications, Springer Healthcare, UK (supported by Merck KGaA, Darmstadt, Germany), for assistance with the preparation of this manuscript.

\section{Funding}

This study was supported by Merck, Switzerland, an affiliate of Merck KGaA, Darmstadt, Germany.

Availability of data and materials

Data are available on request.

\section{Authors' contributions}

ACM contributed to the study design, acted as an international coordinator of the study and participated in data analysis and interpretation and in writing of the manuscript. $A B$ performed the patient examinations. FE participated in the study as a principal investigator at his study site and was responsible for patient enrolment and coordination. PF contributed to the acquisition, analysis and interpretation of the data and was responsible for the conduct of the study at his study site. CDL contributed to the interpretation of the data. VL was involved in the acquisition, analyses and interpretation of the biochemical data. FR made a substantial contribution to the acquisition, analysis and interpretation of the data. HSS made a substantial contribution to the clinical study. SV contributed to the acquisition of the clinical data, as one of the principal investigators on the study. MOB contributed to all the clinical steps of the study. GG participated as investigator in the clinical trial and provided patient data. RP was responsible for the collection and interpretation of the data from the patients in the Czech Republic. MC was involved in the execution of the study and in the review of the study report. AL-H participated in the collection of patient data. AM contributed to the development of the 
population pharmacokinetic analysis plan and the interpretation of these data. DRM conducted the population PK analysis. FM-S participated in the design of the study and performed the statistical analysis. DR contributed to the review of the data. All authors were involved in the writing, revision and final approval of the manuscript of the manuscript.

\section{Competing interests}

ACM has participated in strategic advisory boards and has received honoraria as a consultant and as a speaker from Merck KGaA, Darmstadt, Germany. GG has received support for travel expenses to the SSIEM congress from Merck. FR has participated in advisory boards and has received honoraria as a consultant from Merck KGaA, Darmstadt, Germany. Both DR and FM-S are employees of EMD Serono, Inc, Billerica, MA, USA, a business of Merck KGaA, Darmstadt, Germany. AM is an employee of the Merck Institute for Pharmacometrics, Lausanne, Switzerland, a subsidiary of Merck KGaA, Darmstadt, Germany. AB is an advisory board member for Danone and Merck. PF has received grants from Merck. CDL has received travel fees and provided data management support. MC has been an advisory board member for, and her institution has received a grant from, Merck. A L-H has received travel grants from Merck. DRM was also a paid consultant of Merck KGaA, Darmstadt, Germany. All authors have received fees as an investigator for Merck KGaA, Darmstadt, Germany.

\section{Consent for publication}

Consent to publish has been obtained from all parents and guardians.

\section{Ethics approval and consent to participate}

The local ethics committee/institutional review board at each of the participating centres approved the protocol. Patients' parent(s)/guardian(s) gave written informed consent for participation in the study before any trial-related procedures were performed. Ethics committee: Lead Ethics Committee of the LMU, Munich, Germany (ethikkommission.med.uni-muenchen.de).

\section{Author details \\ ${ }^{1}$ University Children's Hospital, University Medical Center Hamburg Eppendorf, Martinistrasse 52, D-20246 Hamburg, Germany. ${ }^{2}$ University Hospital, Padova, Italy. ${ }^{3}$ Universitair Ziekenhuis Antwerpen, Antwerp, Belgium. ${ }^{4}$ Children's Hospital Kreiskliniken, Reutlingen, Germany. ${ }^{5}$ Hôpital Universitaire des Enfants Reine Fabiola, Brussels, Belgium. ' Universita La Sapienza, Rome, Italy. ${ }^{7}$ Muenster University Children's Hospital, Muenster, Germany. ${ }^{8}$ Hacettepe University School of Medicine, Ankara, Turkey. ${ }^{9}$ Birmingham Children's Hospital, Birmingham, UK. ${ }^{10}$ Department of Pediatrics, University of Bologna, Bologna, Italy. ${ }^{11}$ Centre for Paediatric and Adolescent Medicine, Division for Neuropaediatrics and Metabolic Medicine, University of Heidelberg, Heidelberg, Germany. ${ }^{12}$ University Children's Hospital, Prague, Czech Republic. ${ }^{13}$ Great Ormond Street Hospital, London, UK. ${ }^{14}$ Dr. von Hauner Children's Hospital, Munich, Germany. ${ }^{15}$ Merck Institute for Pharmacometrics, Lausanne, Switzerland. ${ }^{16}$ Projections Research Inc., Phoenixville, USA. ${ }^{17}$ EMD Serono, Billerica, MA, USA.}

\section{Received: 5 August 2016 Accepted: 23 February 2017}

\section{Published online: 09 March 2017}

\section{References}

1. Blau N, Barnes I, Dhondt JL. International database of tetrahydrobiopterin deficiencies. J Inherit Metab Dis. 1996;19:8-14.

2. de Baulny HO, Abadie V, Feillet F, de Parscau L. Management of phenylketonuria and hyperphenylalaninemia. J Nutr. 2007;137:1561S-3S; discussion 73S-75S.

3. Blau N, Hennermann JB, Langenbeck U, Lichter-Konecki U. Diagnosis, classification, and genetics of phenylketonuria and tetrahydrobiopterin (BH4) deficiencies. Mol Genet Metab. 2011;104:S2-9.

4. Scriver CRB AL, Sly WS, Valle D, Vogelstein B, Childs B. The hyperphenylalaninemias. In: The metabolic and molecular bases of inherited diseases. New York: McGraw-Hill Medical; 1995.

5. Blau N, van Spronsen FJ, Levy HL. Phenylketonuria. Lancet. 2010;376:1417-27.

6. Camp KM, et al. Phenylketonuria scientific review conference: state of the science and future research needs. Mol Genet Metab. 2014;112:87-122.

7. van Spronsen FJ, van Rijn M, Bekhof J, Koch R, Smit PG. Phenylketonuria: tyrosine supplementation in phenylalanine-restricted diets. Am J Clin Nutr. 2001;73:153-7
8. Report of Medical Research Council Working Party on Phenylketonuria. Recommendations on the dietary management of phenylketonuria. Arch Dis Child. 1993;68:426-7.

9. Abadie $\mathrm{V}$, et al. Management of phenylketonuria and hyperphenylalaninemia: the French guidelines. Arch Pediatr. 2005;12:594-601.

10. van Spronsen FJ, et al. Key European guidelines for the diagnosis and management of patients with phenylketonuria. Lancet Diabetes Endocrinol. 2017. doi:10.1016/S2213-8587(16)30320-5.

11. Giovannini M, Verduci E, Salvatici E, Paci S, Riva E. Phenylketonuria: nutritional advances and challenges. Nutr Metab. 2012;9:7.

12. Blau N. Sapropterin dihydrochloride for the treatment of hyperphenylalaninemias. Expert Opin Drug Metab Toxicol. 2013;9:1207-18.

13. Sanford M, Keating GM. Sapropterin: a review of its use in the treatment of primary hyperphenylalaninaemia. Drugs. 2009;69:461-76.

14. Qi YL, Mould DR, Zhou HY, Merilainen M, Musson DG. A prospective population pharmacokinetic analysis of sapropterin dihydrochloride in infants and young children with phenylketonuria. Clin Pharmacokinet. 2015; 54:195-207.

15. Duffull SB, Waterhouse TH, Eccleston JE. Some considerations on the design of population pharmacokinetic studies. J Pharmacokinet Pharmacodyn. 2006:32:441-5.

16. Anderson BJ, Allegaert K, Van den Anker JN, Cossey V, Holford NHG. Vancomycin pharmacokinetics in preterm neonates and the prediction of adult clearance. Br J Clin Pharmacol. 2006;63:75-84.

17. Hayton WL. Maturation and growth of renal function: dosing renally cleared drugs in children. AAPS Pharm Sci. 2002;2:1-7.

18. Feillet $F$, et al. Pharmacokinetics of sapropterin in patients with phenylketonuria. Clin Pharmacokinet. 2008;47:817-25.

19. Groselj U, Murko S, Zerjav Tansek M, et al. Comparison of tandem mass spectrometry and amino acid analyzer for phenylalanine and tyrosine monitoring-implications for clinical management of patients with hyperphenylalaninemia. Clin Biochem. 2015;48:14-8.

20. Mo XM, Li Y, Tang AG, Ren YP. Simultaneous determination of phenylalanine and tyrosine in peripheral capillary blood by HPLC with ultraviolet detection. Clin Biochem. 2013;46:1074-8.

21. Gregory CO, Yu C, Singh RH. Blood phenylalanine monitoring for dietary compliance among patients with phenylketonuria: comparison of methods. Genet Med. 2007;9:761-5.

22. Smith I, Beasley MG, Ades AE. Intelligence and quality of dietary treatment in phenylketonuria. Arch Dis Childhood. 1990;65:472-8.

23. Trefz FK, et al. Efficacy of sapropterin dihydrochloride in increasing phenylalanine tolerance in children with phenylketonuria: a phase III, randomized, double-blind, placebo-controlled study. J Pediatr. 2009;154:700-7.

24. Longo $\mathrm{N}$, et al. Long-term developmental progression in infants and young children taking sapropterin for phenylketonuria: a two-year analysis of safety and efficacy. Genet Med. 2015;17:365-73.

25. Shintaku H, Ohura T. Sapropterin is safe and effective in patients less than 4-years-old with BH4-responsive phenylalanine hydroxylase deficiency. J Pediatr. 2014;165:1241-4.

26. Burlina A, Blau N. Effect of BH(4) supplementation on phenylalanine tolerance. J Inherit Metab Dis. 2009;32:40-5.

27. Weglage J, et al. Normal clinical outcome in untreated subjects with mild hyperphenylalaninemia. Pediatr Res. 2001;49:532-6.

28. MacLeod EL, Gleason ST, van Calcar SC, Ney DM. Reassessment of phenylalanine tolerance in adults with phenylketonuria is needed as body mass changes. Mol Genet Metab. 2009;98:331-7.

\section{Submit your next manuscript to BioMed Central and we will help you at every step:}

- We accept pre-submission inquiries

- Our selector tool helps you to find the most relevant journal

- We provide round the clock customer support

- Convenient online submission

- Thorough peer review

- Inclusion in PubMed and all major indexing services

- Maximum visibility for your research

Submit your manuscript at www.biomedcentral.com/submit 